\title{
Fourier Inversion of the Mojette Transform
}

\author{
Andrew Kingston ${ }^{1}$, Heyang $\mathrm{Li}^{1}$, Nicolas Normand ${ }^{2}$, and Imants Svalbe ${ }^{3}$ \\ 1 Dept. Applied Maths, RSPE, The Australian National University, \\ Canberra ACT 2600, Australia \\ andrew.kingston@anu.edu.au \\ 2 IRCCyN, École Polytechnique de l'Université de Nantes, \\ La Chantrerie, Nantes 44306, France \\ 3 School of Physics, Monash University, Clayton VIC 3800, Australia
}

\begin{abstract}
The Mojette transform is a form of discrete Radon transform that maps a $2 \mathrm{D}$ image $(P \times Q$ pixels $)$ to a set of $I$ 1D projections. Several fast inversion methods exist that require $\mathrm{O}(P Q I)$ operations but those methods are ill-conditioned. Several robust (or well-conditioned) inversion methods exist, but they are slow, requiring $\mathrm{O}\left(P^{2} Q^{2} I\right)$ operations. Ideally we require an inversion scheme that is both fast and robust to deal with noisy projections. Noisy projection data can arise from data that is corrupted in storage or by errors in data transmission, quantisation errors in image compression, or through noisy acquisition of physical projections, such as in X-ray computed tomography. This paper presents a robust reconstruction method, performed in the Fourier domain, that requires $\mathrm{O}\left(P^{2} Q \log P\right)$ operations.
\end{abstract}

Keywords: Radon transform, Mojette transform, Fourier inversion, tomography.

\section{Introduction}

The Mojette transform is a discrete form of the Radon transform that maps a $2 \mathrm{D}$ image $(P \times Q$ pixels $)$ to a set of $I$ 1D projections. It was first developed by Guedon et al in 1996 [5] in the context of psychovisual image coding. Due to its distribution, redundancy, and invertibility properies, it has since found applications in distributed data storage on disks and networks, network packet data transmission, tomographic reconstruction, image watermarking, image compression, and image encryption. A summary of the early development of the Mojette transform was the subject of an invited paper to DGCI in 2005 [6] and a book on the Mojette transform was published in 2009 [4].

The Radon transform has many inversion algorithms based on the form of the input data and the a priori information available. Similarly, the Mojette transform has many inverse algorithms. There are fast methods that use local back-projection (LBP) [10] and geometrically driven LBP (GLBP) [11]. These recover the original data in $\mathrm{O}(P Q I)$ operations, however, they are ill-conditioned being essentially forms of Gaussian elimination; any errors in the determination of a variable value are propagated and compounded throughout the remaining 
variables. There are also well-conditioned, or robust, methods that have been explored in the context of tomographic reconstruction. These include a conjugate gradient back-projection (CGBP) 12 that requires $\mathrm{O}\left(P^{2} Q^{2} I\right)$ operations, and an exact back-projection (EBP) method that requires an inordinate number of projections $\left(I \approx 12 P Q / \pi^{2}\right.$ projections) and therefore $\mathrm{O}\left(P^{2} Q^{2}\right)$ operations.

To date there has been no Mojette inversion approach that is fast, exact given noise-free projections, and well-conditioned given noisy projections. This paper presents an inversion algorithm based on the classic Fourier inversion (FI) technique for the Radon transform. It solves rows of Fourier space using a conjugate gradient algorithm that minimises the L2 norm of the error (arising from noise). Assuming $I$ is $\mathrm{O}(P)$, Mojette FI is slower than LBP and GLBP requiring $\mathrm{O}\left(P^{2} Q \log P\right)$ operations but more robust and is faster than CGBP and EBP but less robust.

The remainder of the paper is outlined as follows: Section 2 defines the Mojette transform and the requirements for a unique reconstruction (in the noise-free case). The Fourier properties of the Mojette projection data are outlined in Section 3. The Fourier inversion based on these properties is developed in Section 4. Some results are presented in Section 5 followed by some concluding remarks in Section 6 .

\section{The Mojette Transform}

The Mojette transform of a $2 \mathrm{D}$ function, $f(k, l)$, is comprised of a set of $I$ projections. Each projection has an associated projection direction vector, $\left(p_{i}, q_{i}\right)$, and is comprised of a set of parallel discrete sums over $f$ along lines defined by this vector, i.e., $b=k q_{i}-l p_{i}$. The value of the Mojette transform in each bin, $b$, of a projection is then defined as follows:

$$
\operatorname{proj}_{p_{i}, q_{i}}(b)=\sum_{k=-P / 2}^{P / 2} \sum_{l=-Q / 2}^{Q / 2} f(k, l) \delta\left(b-k q_{i}+l p_{i}\right),
$$

where $\delta(x)=1$ if $x=0$ and is 0 otherwise. Note that $p_{i} \in \mathbb{Z}$, and $q_{i} \in \mathbb{N}$ such that $\operatorname{gcd}\left(p_{i}, q_{i}\right)=1$. The distance between adjacent parallel lines (or sampling rate) of a projection varies with the direction vector, $\left(p_{i}, q_{i}\right)$, as $1 / \sqrt{p_{i}^{2}+q_{i}^{2}}$. The number of bins per projection also varies with $\left(p_{i}, q_{i}\right)$, as follows:

$$
B_{i}=\left|p_{i}\right| Q+q_{i} P
$$

The set of $I$ projections is arbitrary both in cardinality and direction vectors. Therefore, a criterion is required to determine when a sufficient number of projections have been acquired in order to ensure a unique solution for a $P \times Q$ dataset. Katz determined the following criterion [7]:

$$
\sum_{i=0}^{I-1}\left|p_{i}\right| \geq P \quad \text { or } \quad \sum_{i=0}^{I-1} q_{i} \geq Q
$$


For the remainder of this paper we will assume that $\sum\left|p_{i}\right| \geq P$. Below Katz criterion there is no unique solution, therfore the condition number is infinite; when Katz criterion is just satisfied, i.e., $\sum\left|p_{i}\right|=P$, the condition number is very large so any noise is amplified and propagated into the reconstruction; as additional projections are added, the condition number decreases rapidly at first but then with diminishing returns.

\section{Fourier Properties of Mojette Projections}

Before developing a Fourier inversion technique it is beneficial to establish how Mojette projection data maps into discrete Fourier space. First we define the 1D discrete Fourier transform (DFT) of $g(k)$ as follows:

$$
\mathcal{F}^{1}\{g\}=\widehat{g}(u)=\sum_{k=-P / 2}^{P / 2} g(k) \exp (-i 2 \pi k u / P),
$$

and define the 2D DFT of $g(k, l)$ as follows:

$$
\mathcal{F}^{2}\{g\}=\widehat{g}(u, v)=\sum_{k=-P / 2}^{P / 2} \sum_{l=-Q / 2}^{Q / 2} g(k, l) \exp (-i 2 \pi k u / P) \exp (-i 2 \pi l v / Q) .
$$

A discrete form of the Fourier slice theorem for classical Radon projections was first presented in 3 using the Z-transform. It was first applied to the $n \mathrm{D}$ Mojette transform by Verbert and Guédon in [13] also using the Z-transform. Here we demonstrate it for the 2D Mojette transform using the DFT.

Theorem 1. The $1 D$ DFT of a Mojette projection of $f$ maps to a discrete line (or "slice") through the origin of the 2D DFT of $f$ as follows:

$$
\mathcal{F}^{1}\left\{\operatorname{proj}_{p_{i}, q_{i}}\right\}(w)=\widehat{f}\left(\frac{q_{i} P}{B_{i}} w, \frac{-p_{i} Q}{B_{i}} w\right)
$$

Proof.

$$
\begin{aligned}
\mathcal{F}^{1}\left\{\operatorname{proj}_{p_{i}, q_{i}}\right\} & =\sum_{b=-B_{i} / 2}^{B_{i} / 2} \sum_{k=-P / 2}^{P / 2} \sum_{l=-Q / 2}^{Q / 2} f(k, l) \delta\left(b-k q_{i}+l p_{i}\right) \exp \left(-i 2 \pi b w / B_{i}\right) \\
& =\sum_{k=-P / 2}^{P / 2} \sum_{l=-Q / 2}^{Q / 2} f(k, l) \exp \left(-i 2 \pi\left(k q_{i}-l p_{i}\right) w / B_{i}\right) \\
& =\sum_{k=-P / 2}^{P / 2} \sum_{l=-Q / 2}^{Q / 2} f(k, l) \exp \left(-i 2 \pi \frac{k}{P}\left(q_{i} P w\right) / B_{i}\right) \\
& =\widehat{f}\left(\frac{q_{i} P}{B_{i}} w, \frac{-p_{i} Q}{B_{i}} w\right)
\end{aligned}
$$

Figure 1 provides a depiction of the theorem. The sampling rate of the DFT of projection data in frequency space is $\sqrt{q_{i}^{2} P^{2}+p_{i}^{2} Q^{2}} / B$. An important property of the slices that arises is the following: 


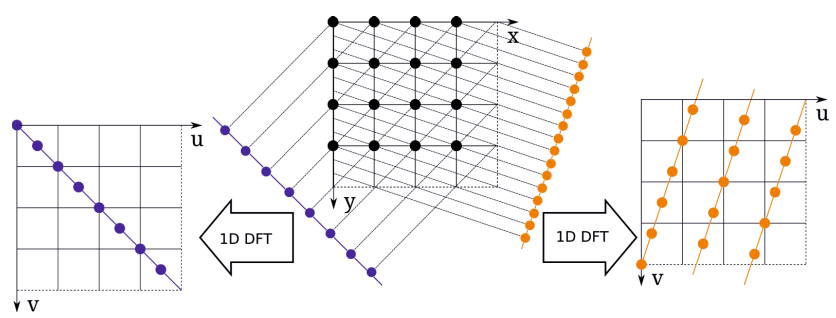

Fig. 1. A depiction of Theorem 1 for $\operatorname{proj}_{-1,1}$ and $\operatorname{proj}_{3,1}$ on a $4 \times 4$ image

Lemma 2. Given the slice in Fourier space formed from a projection with direction vector $\left(p_{i}, q_{i}\right)$, any horizontal line, $v=$ const, intersects the slice exactly $\left|p_{i}\right|$ times and any vertical line, $u=\mathrm{const}$, intersects the slice exactly $q_{i}$ times.

Proof. We have $w \in \mathbb{Z}_{B_{i}}$ and from Theorem 1 the sampling step between each successive point in frequency space is $\left(\frac{q_{i} P}{B_{i}}, \frac{-p_{i} Q}{B_{i}}\right)$. Therefore, the total sampling displacement in frequencey space is $\left(\frac{q_{i} P}{B_{i}}, \frac{-p_{i} Q}{B_{i}}\right) B_{i}=\left(q_{i} P,-p_{i} Q\right) \equiv(0,0)$ mod $(P, Q)$. There is no overlap in the sampling since $p_{i}$ and $q_{i}$ are coprime, hence implying the theorem.

\section{Mojette Fourier Inversion}

The frequency data from the $I$ slices gives us a nonuniform sampling of discrete Fourier space. Before continuing, we shall introduce the nonuniform Fourier transform (NFT). We define the 1D FT of irregularly sampled data to $J$ frequencies as:

$$
\widehat{g}\left(w_{j}\right)=\sum_{k=-N / 2}^{N / 2} g(k) \exp \left(-i 2 \pi k w_{j}\right)
$$

for $w_{j} \in[-0.5,0.5)$ and $j \in \mathbb{Z}_{J}$ The NFT can be performed in $\mathrm{O}(N \log N)$ operations, see [8]. Iterative inversion of the NFT can be achieved through the conjugate gradient method which converges within $N$ iterations. Therefore, the inverse NFT (INFT) can be obtained in $\mathrm{O}\left(N^{2} \log N\right)$ operations. In practice this can be much faster if the right pre-conditioner is used, see 9 .

Robust Mojette Fourier inversion can of course be achieved by taking the frequency data from the $I$ slices and performing a 2D INFT. However, this will be performed in $\mathrm{O}\left(P^{2} Q^{2} \log P Q\right)$ operations. This has been done for projections with $5 \%$ noise for comparison with the proposed method in sect. 5. In what follows we describe a method to achieve a less robust inversion in only $\mathrm{O}\left(P^{2} Q \log Q\right)$ operations by a deliberate sampling of the slice data to lie on horizontal lines enabling the 1D INFT to be applied to each line followed by an inverse fast Fourier transform (IFFT) applied to the columns. 


\subsection{Exact Resampling of Projection Slices}

The slice data can be exactly resampled to lie on common horizontal lines in 2D discrete Fourier space, similar to [3]13 and Theorem 1] but using a specific Chirp z-transform of projections. First let us define the 1D Chirp z-transform as follows:

$$
\mathcal{Z}^{1}\{g\}=\widetilde{g}(w)=\sum_{k=-N / 2}^{N / 2} g(k) z^{w k}, \quad w \in \mathbb{Z}_{M} .
$$

Here $z \in \mathbb{C}$ and typically $|z|=1$. As was done by Bailey and Swarztrauber in [1] we define $z=\exp (-i 2 \pi \alpha)$ for $w \in \mathbb{Z}$ and $\alpha \in \mathbb{R}$ to give:

$$
\mathcal{Z}_{\alpha}^{1}\{g\}=\widehat{g}^{\alpha}(w)=\sum_{k=-N / 2}^{N / 2-1} g(k) \exp (-i 2 \pi k w \alpha)=\widehat{g}(N \alpha w) .
$$

So $\alpha=1 / N$ gives the frequency data of the $1 \mathrm{D}$ DFT. This can be done in $\mathrm{O}(N \log N)$ if $\alpha$ is rational, (see [2]1]). Here we use $\alpha=1 /\left(\left|p_{i}\right| Q\right)$ so that the frequency sampling rate is 1.0 in the $v$-direction of $\widehat{f}(u, v)$ as follows:

\section{Corollary 3.}

$$
\mathcal{Z}_{\frac{1}{\left|p_{i}\right| Q}}^{1}\left\{\operatorname{proj}_{p_{i}, q_{i}}\right\}(w)=\widehat{f}\left(\frac{q_{i} P}{\left|p_{i}\right| Q} w,-\operatorname{sgn}\left(p_{i}\right) w\right)
$$

Proof.

$$
\begin{aligned}
& \mathcal{Z}_{\frac{1}{\left|p_{i}\right| Q}}^{1}\left\{\operatorname{proj}_{p_{i}, q_{i}}\right\}(w)=\sum_{b=-B_{i} / 2}^{B_{i} / 2} \sum_{k=-P / 2}^{P / 2-1} \sum_{l=-Q / 2}^{Q / 2-1} f(k, l) \delta\left(b-k p_{i}+l p_{i}\right) \\
& \exp \left(-i 2 \pi b w /\left|p_{i}\right| Q\right) \\
& =\sum_{k=-P / 2}^{P / 2-1} \sum_{l=-Q / 2}^{Q / 2-1} f(k, l) \exp \left(-i 2 \pi\left(k q_{i}-l p_{i}\right) w /\left|p_{i}\right| Q\right) \\
& =\sum_{k=-P / 2}^{P / 2-1} \sum_{l=-Q / 2}^{Q / 2-1} f(k, l) \exp \left(-i 2 \pi \frac{k}{P}\left(q_{i} P w\right) /\left|p_{i}\right| Q\right) \\
& =\widehat{f}\left(\frac{q_{i} P}{\left|p_{i}\right| Q} w,-\operatorname{sgn}\left(p_{i}\right) w\right) \\
& \exp \left(-i 2 \pi \frac{l}{Q}-\operatorname{sgn}\left(p_{i}\right) w\right)
\end{aligned}
$$

From Lemma 2 there are $\left|p_{i}\right|$ intersections with any horizontal line, thus each slice samples $\left|p_{i}\right| Q$ frequencies in $\widehat{f}$. This causes all frequency data from the slices to lie on lines $v=c$ for $c \in \mathbb{Z}_{Q}$ and all projection slice data combined samples $\sum\left|p_{i}\right| Q \geq P Q$ frequencies provided each sample point is unique. The problem is that at $v=0$ all lines intersect giving redundant data, hence insufficient unique data for exact inversion. This can be overcome by offsetting the sampling in the $v$-direction by a fraction $\phi \in(0,1)$. An offset of frequency data by $\phi$ can of course be done as a phase shift on the projections prior to applying the $1 \mathrm{D}$ Chirp z-transform. An example of the resulting data is depicted in Fig. 2 
(a)

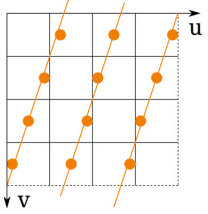

(b)

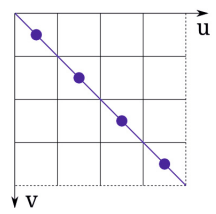

(c)

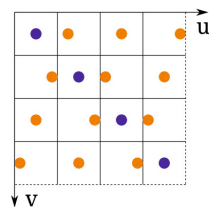

Fig. 2. A depiction of the chirp z-transforms of (a) $\operatorname{proj}_{-1,1}$ and (b) $\operatorname{proj}_{3,1}$ on a $4 \times 4$ image with $\phi=0.5$. $\alpha$ is selected to give data on $v \in\{-1.5,-0.5,0.5,1.5\}$ frequencies. (c) Results from (a) and (b) overlayed in the Fourier domain; note there are $\sum_{I}\left|p_{i}\right|$ samples in each row and $Q$ rows of samples. According to Katz criterion (3) this is a reconstructible sample set.

Ideally we would set $\phi=0.5$ for our slice frequency data points to be as far from the origin as possible. However slices may intersect at points other than the origin. We need to check if the frequency data points sampled by the slices intersect at $v=c+\phi$ for $c \in \mathbb{Z}_{Q}$. We must identify some sufficient conditions to ensure no overlapping data points in Fourier space. Using the result from Corollary [3, the coordinate in fourier space for the projection with a direction vector $\left(p_{i}, q_{i}\right)$ on a $P \times Q$ image with phaseshift of $\phi$ is:

$$
(c+\phi)\left(q_{i} P / p_{i} Q, 1\right) \bmod (P, Q), \quad \text { for } c \in\left[0, q_{i} P-1\right] .
$$

In order for two Fourier data points to meet, they must come from two different projections. Let these two projections have direction vectors $\left(p_{1}, q_{1}\right)$ and $\left(p_{2}, q_{2}\right)$ with $c=c_{1}$ and $c_{2}$ respectively. Placing this into (11) gives the following:

$$
\left(c_{1}+\phi\right)\left(q_{1} P / p_{1} Q, 1\right)=\left(c_{2}+\phi\right)\left(q_{2} P / p_{2} Q, 1\right) \bmod (P, Q),
$$

for $c_{1} \in \mathbb{Z}_{p_{1} Q}$ and $c_{2} \in \mathbb{Z}_{p_{2} Q}$. This equation implies $c_{1}=c_{2} \bmod (Q)$ and $\left(c_{1}+\phi\right)\left(q_{1} P / p_{1} Q\right)=\left(c_{2}+\phi\right)\left(q_{2} P / p_{2} Q\right) \bmod (P)$. Therefore we have

$$
\left(c_{1}+\phi\right)\left(\frac{q_{1}}{p_{1} Q}\right)-\left(c_{2}+\phi\right)\left(\frac{q_{2}}{p_{2} Q}\right) \quad \text { is an integer. }
$$

This becomes:

$$
x / p_{1} p_{2} Q+\phi \frac{q_{1} p_{2}-q_{2} p_{1}}{p_{1} p_{2} Q} \text { for } \quad x \in \mathbb{Z} .
$$

We can now present a lemma that guarantees no point of intersection:

Proposition 4. Setting $\phi=\frac{k}{2 \cdot k+1}$ with $k=\max _{i, j}\left|p_{i} q_{j}\right|$ would have no point of intersection between two different projection set in Fourier space.

Proof. Setting $\phi=\frac{k}{2 \cdot k+1}$ with $k \geq \max _{i, j}\left|p_{i} q_{j}\right|$ forces $q_{1} p_{2}-q_{2} p_{1}=0$ as otherwise (13) is false. Since the $\operatorname{gcd}\left(p_{i}, q_{i}\right)=\operatorname{gcd}\left(p_{j}, q_{j}\right)=1$, we have $p_{i}=p_{j}$ and $q_{i}=q_{j}$ implies there is no point of intersection in Fourier space. With this shift, we can also guarantee a minimum seperation in Fourier space of $\min _{i, j} \frac{P}{Q p_{i} p_{j}(2 k+1)}$ with $i \neq j$.

Placing the offset, resampled, slice frequency data from each projection into 2D Fourier space now gives us data as depicted in Fig. 2 r. 


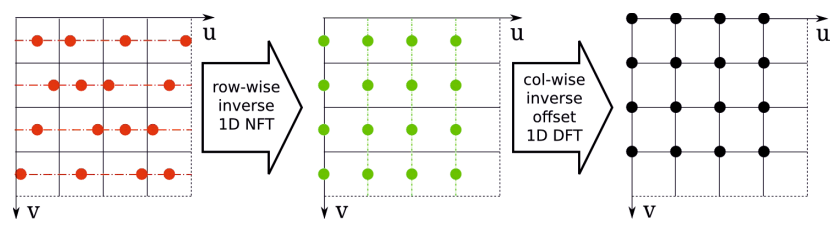

Fig. 3. A depiction of the process to reconstruct a $4 \times 4$ image from the chirp $z^{-}$ transforms of proj- $1,1_{1}$ and $\operatorname{proj}_{3,1}$

\subsection{Reconstruction Process}

This section outlines the Fourier inversion process, as depicted in Fig. 3. to recover $f$ from the offset, resampled, slice frequency data, e.g., Fig. 22. First we propose the following theorem:

Theorem 5. For a set of Mojette projections that satisfies Katz Criterion (3), there is sufficient slice data to be inverted in Fourier space after the chirp Ztransform with $\alpha=1 /\left(\left|p_{i}\right| Q\right)$ and no intersecting data points.

Proof. Here we are assuming Katz Criterion is satisfied by $\sum\left|p_{i}\right| \geq P$. By Lemma 2 and Corollary 3 the Chirp z-transform of all projections gives $Q$ rows of data points with $\sum\left|p_{i}\right| \geq P$ elements in each row. Since none of these points intersect, each row is invertible using the 1D INFT. There are $Q$ data points in each column that are invertible using the 1D IDFT.

Proposition 4 guarantees no intersecting data points in Fourier space with particular choice of phase shift, $\phi$. Therefore we can satisfy the requirement of no intersecting data points in Theorem 5 .

The entire reconstruction process, given I Mojette projections, can be summarised in the following 6 steps:

1. Calculate $\phi=\frac{k}{2 \cdot k+1}$ with $k=\max _{i, j}\left|p_{i} q_{j}\right|$;

2. Apply the phase shift, $\phi$, to all projection data;

3. Fast chirp z-transform each projection with $\alpha=1 /\left(\left|p_{i}\right| Q\right)$;

4. Remap frequency data to 2D Fourier domain;

5. Invert nonuniform frequency data in rows. $Q$ rows $\times$ maximum $P$ iterations of INFT.

6. Inverse Fast Fourier transform of $P$ columns (with a phase shift of $-\phi$ );

\section{Results}

In this section we demonstrate the performance of the proposed algorithm with various noise conditions and various number and distribution of projection direction vectors. We investigate $0 \%, 1 \%$ and $5 \%$ Gaussian noise added to the projection data, i.e., noise following a normal distribution with a standard deviation equal to $\eta \%$ of the average value. We also investigate three types of angle 
distributions, $F_{N}^{180}, F_{N}^{90}, M_{N}$. Here, $F_{N}$ denotes the direction vectors formed by the Farey series, e.g., $F_{3}=0 / 1,1 / 3,1 / 2,2 / 3,1 / 1$ corresponds to direction vectors $(1,0),(3,1),(2,1),(3,2),(1,1)$. The 90 and 180 signify 90 degree or 180 degree symmetries, e.g., for direction vector $(a, b), 90$ includes $(-a, b)$ while 180 also includes $(b, a)$ and $(-b, a) . M_{N}$ denotes the set of direction vectors $(1,0),(1,1),(-1,1),(2,1),(-2,1), \ldots,(N, 1),(-N, 1)$. All simulations are for a $256 \times 256$ image of Lena shown in Fig. 4 a

(a)

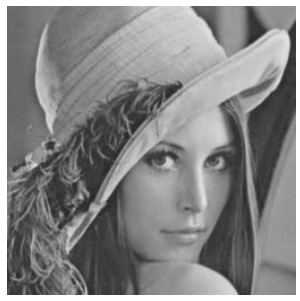

(b) $5 \%$ noise

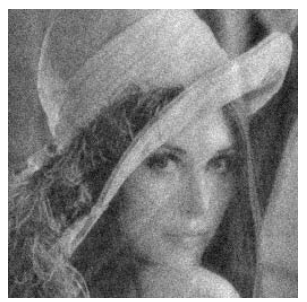

Fig. 4. (a) original image used for simulations. (b) Reconstruction using full 2D-MFI for Mojette data with angle set $F_{8}^{180}$ and $5 \%$ Gaussian noise added. RMSE 17.1.

Firstly, using the $F_{8}^{180}$ angle set we investigated the effect of $0 \%, 1 \%$, and $5 \%$ noise added to the projections. The results are presented in Fig. 57. It can be seen that the proposed method can deal with a moderate amount of noise. It is not as robust as CGM (see Fig. 4b); the RMSE data shows that noise is amplified in the reconstruction. Even the noise-free input data has a non-zero RMSE. This seems to be due to round-off errors, paricularly a problem for the INFT of rows $v=\phi$ and $v=\phi-1$ of Fourier space. Smaller arrays with $P, Q \leq 32$ do not suffer from this.

Secondly, by adding a fixed level of $1 \%$ noise to the projection data, we establish the effect of the number of projections, and more specifically the value of $\sum_{i} p_{i}$ as it increases above Katz limit (3). Figure $5 \mathrm{~b}$ gives the reconstructions using $F_{7}^{180}, F_{8}^{180}$, and $F_{9}^{180}$ with $\sum_{i} p_{i}=275,371$, and 533 respectively. As expected, the results improve as $\sum_{i} p_{i}$ increases and the condition number of the system decreases.

Lastly, by again adding $1 \%$ noise to the projection data, we explore the effect of angle distribution. We use three angle sets that each have a similar $\sum_{i} p_{i}, F_{9 a}^{90}$, $F_{7}^{180}$, and $M_{16}$ with $\sum_{i} p_{i}=283,275$, and 273 respectively. (Note 9 a indicates we are using $F_{8}^{90}$ and adding only direction vectors $( \pm 9,1)$ and $( \pm 9,2)$ from $\left.F_{9}\right)$. With $\sum_{i} p_{i}$ very close to Katz limit (3) this is very unstable (as described in sect. 2). Results are presented in Fig. 56 and show that reconstruction with a more symmetric angle set with greater redundancy performs better than limited angle datasets. 
(a-i) $0 \%$ noise

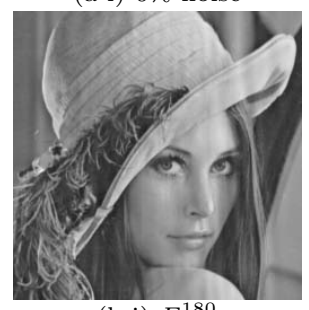

(b-i) $F_{7}^{180}$

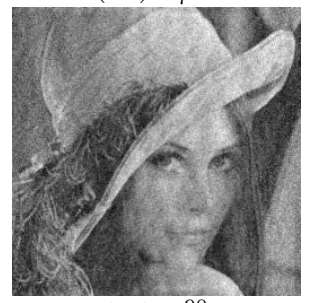

(c-i) $F_{9 a}^{90}$

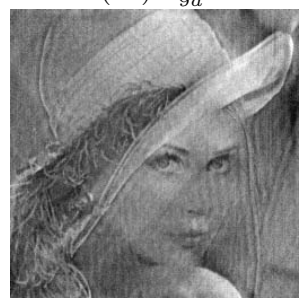

(a-ii) $1 \%$ noise

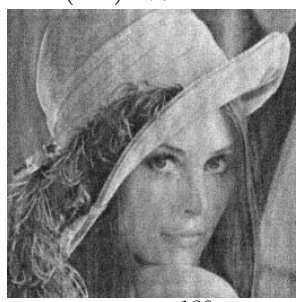

(b-ii) $F_{8}^{180}$

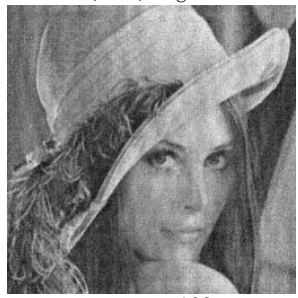

(c-ii) $F_{7}^{180}$

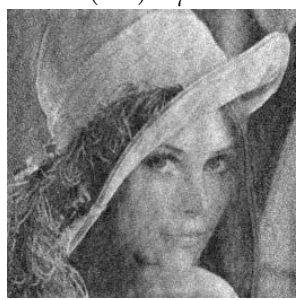

(a-iii) $5 \%$ noise

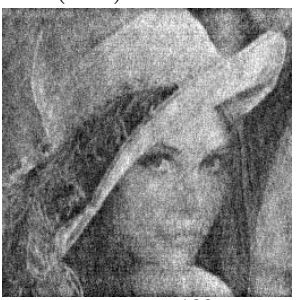

(b-iii) $F_{9}^{180}$

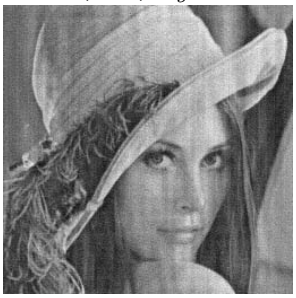

(c-iii) $M_{16}$

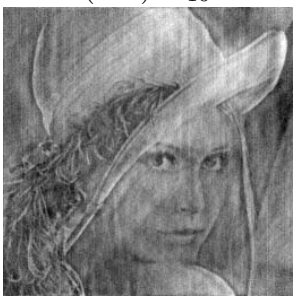

Fig. 5. (a) Reconstruction using MFI for Mojette data with angle set $F_{8}^{180}$. Amount of Gaussian noise added as indicated. RMSE (i) 6.0 (ii) 16.2 (iii) 26.7. (b) Reconstruction using MFI for Mojette data with 1\% Gaussian noise added using angle set as indicated. RMSE (i) 19.5 (ii) 16.2 (iii) 13.1. (c) Reconstruction using MFI for Mojette data with $1 \%$ Gaussian noise added using angle set as indicated. RMSE (i) 28.3 (ii) 19.5 (iii) 29.1 .

\section{Conclusion}

We have presented a reconstruction technique for Mojette projection data that is based on the Fourier inversion technique for the classical Radon transform. We use exact frequency data resampling to make the slice data lie on a set of parallel lines in Fourier space. This enables inversion to be broken into a set of 1D INFT and a set of 1D IFFT and speeds up the process. Given the Mojette transform data of a $P \times Q$ image with $I$ projections, our Fourier inversion algorithm requires $\mathrm{O}\left(P^{2} Q \log P\right)$ operations. This technique is a compromise between fast, $\mathrm{O}(P Q I)$, but ill-conditioned local back-projection algorithms and slow, $\mathrm{O}\left(P^{2} Q^{2} I\right)$ but well-conditioned techniques. Results show that this method can tolerate a moderate amount of noise and that redundant and symmetrical projection data is preferable for increased performance. Future work includes 
determining a stopping criterion for the INFT and investigating regularisation or weighting techniques to make MFI more robust.

Acknowledgements. AK acknowledges l'Univ. de Nantes for funding a one month visit to IVC-IRCCyN where much of this work was undertaken.

\section{References}

1. Bailey, D., Swarztrauber, P.: The fractional fourier transform and applications. SIAM Review 33, 389-404 (1991)

2. Bluestein, L.: A linear filtering approach to the computation of the discrete fourier transform. Northeast Electronics Research and Engineering Meeting Record 10 (1968)

3. Dudgeon, D., Mersereau, R.: Multidimensional Digital Signal Processing. PrenticeHall (1983)

4. Guédon, J.: The Mojette Transform: theory and applications. ISTE-Wiley (2009)

5. Guédon, J., Barba, D., Burger, N.: Psychovisual image coding via an exact discrete Radon transform. In: Wu, L.T. (ed.) Proceedings of Visual Communication and Image Processing 1995, pp. 562-572 (May 1995)

6. Guédon, J., Normand, N.: The Mojette transform: the first ten years. In: Andrès, É., Damiand, G., Lienhardt, P. (eds.) DGCI 2005. LNCS, vol. 3429, pp. 79-91. Springer, Heidelberg (2005)

7. Katz, M.: Questions of uniqueness and resolution in reconstruction from projections. Springer (1977)

8. Keiner, J., Kunis, S., Potts, D.: Using NFFT 3 - a software library for various nonequispaced fast Fourier transforms. ACM Trans. Math. Software 36, 1-30 (Article 19) (2009)

9. Kunis, S., Potts, D.: Stability results for scattered data interpolation by trigonometric polynomials. SIAM J. Sci. Comput. 29, 1403-1419 (2007)

10. Normand, N., Guédon, J., Philippe, O., Barba, D.: Controlled redundancy for image coding and high-speed transmission. In: Ansari, R., Smith, M. (eds.) Proceedings of SPIE Visual Communications and Image Processing 1996, vol. 2727, pp. 1070-1081. SPIE (February 1996)

11. Normand, N., Kingston, A., Évenou, P.: A geometry driven reconstruction algorithm for the Mojette transform. In: Kuba, A., Nyúl, L.G., Palágyi, K. (eds.) DGCI 2006. LNCS, vol. 4245, pp. 122-133. Springer, Heidelberg (2006)

12. Servières, M., Idier, J., Normand, N., Guédon, J.: Conjugate gradient Mojette reconstruction. In: Fitzpatrick, J., Reinhardt, J. (eds.) Proceedings of SPIE Medical Imaging 2005: Image Processing, vol. 5747, pp. 2067-2074 (April 2005)

13. Verbert, P., Guédon, J.: N-dimensional Mojette transfrom. Application to multiple description. IEEE Discrete Signal Processing 2, 1211-1214 (2002) 